\title{
Quantification of Patient and Equipment Handling for Nurses through Direct Observation and Subjective Perceptions
}

\author{
Tiffany Poole Wilson, ${ }^{1}$ Kermit G. Davis, ${ }^{1}$ Susan E. Kotowski, ${ }^{2}$ and Nancy Daraiseh ${ }^{3}$ \\ ${ }^{1}$ Low Back Biomechanics and Workplace Stress Laboratory, University of Cincinnati, 423 Kettering Laboratory Building, \\ 3223 Eden Avenue, Cincinnati, OH 45267-0056, USA \\ ${ }^{2}$ College of Allied Health Sciences, University of Cincinnati, French East Building, 3202 Eden Avenue, \\ Cincinnati, OH 45267-0379, USA \\ ${ }^{3}$ Cincinnati's Children's Medical Center, 3333 Burnet Avenue, Cincinnati, OH 45229-3026, USA
}

Correspondence should be addressed to Kermit G. Davis; kermit.davis@uc.edu

Received 16 September 2014; Accepted 4 December 2014

Academic Editor: Sue Read

Copyright (C) 2015 Tiffany Poole Wilson et al. This is an open access article distributed under the Creative Commons Attribution License, which permits unrestricted use, distribution, and reproduction in any medium, provided the original work is properly cited.

\begin{abstract}
Background. Musculoskeletal disorders have continued to plague nurses in hospitals and long-term care facilities. Low back and shoulder injuries are the most prevalent, frequently linked to patient handling activities. Exposure to patient handling has been predominantly quantified by subjective responses of nurses. Objective. To directly observe handling of patients and other medical equipment for nurses during a 12-hour work shift. Methods. Twenty nurses working in three different intensive care units at a Midwest teaching hospital were directly observed during 12-hour day shifts. Direct observation included documenting frequency and type of handling performed and whether lift assist devices were utilized. Two additional surveys were completed by nurses to assess current pain levels and perceptions of lifting being performed. The observed lifting was compared to the perceived lifting with simple inference statistics. Results. Nurses have a high prevalence of manually lifting patients and medical devices but limited use of lifting assist devices. Nurses handled patients 69 times per shift and medical equipment 6 times per shift, but less than 3\% utilized a lift assist device. Nurses suffered from high levels of pain at the end of the shift, with the highest prevalence in the lower back, lower legs, and feet/ankles (all above 60\%).
\end{abstract}

\section{Introduction}

Musculoskeletal disorders (MSDs) are the most costly workplace injury and the leading cause of occupational injury to nurses [1]. The nursing profession including nurses and nursing aides remains as one of the top industries in reporting MSDs and MSDs with lost days. In 2009, the U.S. Bureau of Labor Statistics reported that the nursing occupation was the industry with the highest MSD incident rate (226 MSD cases per 10,000 full time employees) [1]. The prevalence of work-related back injuries in the nursing profession in the US is $66.8 \%$ with an incidence rate of 90.1 per 10,000 full time hospital nurses [2]. Davis and Kotowski reviewed the literature investigating MSDs in nurses and nursing aides in hospitals and reported that low back pain (54\% prevalence in past year) was the leading injury followed by the shoulders
( $46 \%$ prevalence in past year) and neck $(40 \%$ prevalence in past year) [3]. With so many nurses being adversely impacted by MSDs, the underlying risk factors for these injuries need to be understood.

Prevalence of MSD injuries has been associated with the physically demanding nature of the occupation. Patient handling activities such as transfers and repositioning of patients in bed have been identified as one of the most common causes of MSDs [2, 4-6]. Nurses have reported through surveys that $20 \%$ of their time is spent performing patient handling tasks [7]. The burden of patient handling will likely grow as the population ages and the impact of high obesity rates start to influence patient size. Obviously, heavier patients will place a greater burden on nurses when transferring or repositioning in the bed. However, an interesting trend was found by Randall and associates [8], in that low back injuries were 
lower when transferring bariatric patients, potentially due to higher usage of lift assist devices with these heavy patients.

While patient handling activities have been the primary focal point of MSD research for nurses, other factors could contribute to the high prevalence of MSDs in nursing in hospitals. This short survey listed 15 common patient handling tasks gathered from a previous study which documented the top 10 patient transfer tasks by physical demand $[4,6]$. Some researchers have identified increased risk of MSDs, particularly low back pain with higher rates of handling medical equipment and furniture [9-11]. Thus, the entire handling burden for the nurses must be quantified in order to get a better understanding of why they are getting injured.

Majority of studies investigating handling activities have relied upon subjective assessment of nurses in hospitals. The frequency of handling activities for a shift within the hospital setting has been reported to be repositioning (7.5 to 9.9 times) and transferring (2.8 to 20 times) $[10,12,13]$. Vasiliadou and associates also reported medical equipment transfers of more than 50 times per shift [13]. We found no studies that reported links of patient handling activities to reported MSDs (predominantly low back pain) having used direct observation. Only one study used direct observation to estimate physical demands [6]. The problem with subjective survey assessments is that reality could be drastically different from reported values, biasing the estimation of a relationship between MSDs and risk factors. Only one study was found to have directly observed nurses handling activities in the hospital [6] but this assessment was limited to a 2-hour period. Further, the majority of studies assess patient handling tasks without considering the maneuvering of medical equipment associated with patient care, which may contribute to an increased risk of injury to the nurse. This includes lifting and repositioning monitors, medical assessment tools, and objects of comfort for the patient such as pillows, trays, and personal belongs.

Based on the very limited direct observation of nursing personnel handling patients, medical equipment, and furniture, the purpose of this current study was to objectively quantify patient handling activities performed by nurses during their entire work shift using direct observation and to compare these frequencies to that of perceived handling activities as collected by a survey. The overall study objective was to quantify the frequency of handling tasks performed by nurses in intensive care units.

\section{Methods}

2.1. Study Overview. The study was an observational study that directly observed the handling of patients, medical devices, and furniture in intensive care units (ICU). The research team observed nurses for an entire 12-hour shift and identified the frequency of handling activities. Participants also completed a survey to assess their perceptions of handling activities and current symptoms at the end of the shift. The observations were done at a large Midwest teaching hospital in three units: the medical intensive care unit (MICU), neurosurgical intensive care unit (NSICU), and surgical intensive care unit (SICU).
2.2. Subjects. Twenty nurses participated in the study. All full-time nurses working in the identified hospital units were recruited to participate. Subjects included healthy fulltime registered nurses working 12-hour shifts. Subjects were recruited via posters and in-person requests. In-person requests were made during transition times and specified unit staff meetings on multiple days. Subjects received a nominal valued gift card (\$50) upon the completion of their observation period. A representative sample with respect to gender and age was selected. Study participants signed informed consent documents (approved by the University of Cincinnati's Institutional Review Board), after the study purpose and observational procedures were explained. Selected anthropometric measurements and demographic data are shown in Table 1. Of the 20 nurses who were observed, 10 were from the MICU, 3 from the NSIC, and 7 from the SICU. Study participants were mainly female (70\%) and all worked the day shift. Average years of experience were 6.5 with a little over half (55\%) having 5 years or less. The average age was 34 years old with $65 \%$ of participants under the age of 33 years. The gender and age breakdown are similar to the units observed and the nursing profession.

2.3. Observation Protocol. Nurses were observed during day shifts with observation days being randomly selected across all days of the week. Research team members recorded activities into a spreadsheet accessed on a tablet. Patient handling tasks were preidentified using the 16 stressful tasks identified by Owen and Garg [14]. In addition, the number of times a patient handling activity was performed with lift assist devices was also documented. Finally, handling of medical equipment and furniture in the room was also documented. Every instance of handling was documented during the entire observed shift. At the end of the shift, subjects completed a survey about their perceptions of handling of patients, medical devices, and furniture.

2.4. Questionnaires. A simple questionnaire was completed by all subjects to collect demographic information, health status, and employment history. The questionnaire obtained information about race, ethnicity, gender, age, years as a nurse, years working in current position, education level, height, weight, smoking, and current body discomfort. Participants also rated their current discomfort at the end of the shift. Discomfort was rated on a scale of 0 (no pain) to 10 (most intense pain imaginable) for 9 body areas (neck, shoulders, elbows, hands/wrists, upper back, low back, hips, knees, and feet/ankles). Each of the discomfort ratings was then later classified into four categories $(0=$ None, $1-4=$ Mild, $5-8=$ Moderate, and 9-10 = Severe $)$. The threshold of 1.0 was used to determine the prevalence of discomfort amongst the study participants based on body region.

A short survey on perception of total lifts and transfers performed on a typical day was completed by nurses at the end of the observation period. This short survey listed 15 common patient handling tasks gathered from a previous study which documented the top 10 patient transfer tasks by physical demand [6]. Nurses were requested to provide their 
TABLE 1: Demographic and anthropometry (average and standard deviation) information as a function of intensive care unit and overall.

\begin{tabular}{|c|c|c|c|c|c|c|}
\hline Unit & Gender (male/female) & Age (years) & $\begin{array}{c}\text { Experience in } \\
\text { nursing (years) }\end{array}$ & $\begin{array}{l}\text { Tenure at current } \\
\text { position (years) }\end{array}$ & $\begin{array}{l}\text { Standing height } \\
(\mathrm{cm})\end{array}$ & $\begin{array}{c}\text { Body weight } \\
(\mathrm{kg})\end{array}$ \\
\hline Medical ICU MICU $(N=10)$ & 3 male/7 female & $34.7(12.4)$ & $8.8(10.9)$ & $4.5(1.2)$ & $67.0(4.4)$ & $163.3(50.0)$ \\
\hline Neurosurgical NSICU $(N=3)$ & 1 male $/ 2$ female & $30.3(5.7)$ & $2.5(2.2)$ & $2.5(2.2)$ & $68.3(2.3)$ & $180(50.7)$ \\
\hline Surgical SICU $(N=7)$ & 2 male $/ 5$ female & $33.6(12.1)$ & $4.9(5.1)$ & $2.8(1.9)$ & $66.9(3.7)$ & $166.7(30.7)$ \\
\hline Total $(N=20)$ & 6 male/14 female & $33.7(11.2)$ & $6.5(8.4)$ & $6.0(5.2)$ & $67.2(3.8)$ & $167(42.2)$ \\
\hline
\end{tabular}

TABLE 2: Frequency (number of times per shift) of the patient handling tasks performed by the nurses in the three ICU units and overall as quantified by direct observation and survey (perceived) (average and standard deviation).

\begin{tabular}{|c|c|c|c|c|c|c|c|}
\hline Unit & $\begin{array}{c}\text { Bed to } \\
\text { wheelchair }\end{array}$ & $\begin{array}{l}\text { Bed to } \\
\text { chair }\end{array}$ & $\begin{array}{c}\text { Patient out } \\
\text { of bed }\end{array}$ & $\begin{array}{c}\text { Body } \\
\text { reposition }\end{array}$ & $\begin{array}{c}\text { Bed to assistive } \\
\text { device }\end{array}$ & $\begin{array}{l}\text { In and out } \\
\text { of bathtub }\end{array}$ & $\begin{array}{c}\text { On and off } \\
\text { toilet }\end{array}$ \\
\hline \multicolumn{8}{|c|}{ Direct observation } \\
\hline Medical (MICU) & $0.2(0.6)$ & $1.5(2.0)$ & $2.0(3.2)$ & $35.1(23.5)$ & $0.1(0.3)$ & - & - \\
\hline Neurosurgical (NSICU) & $0.3(0.6)$ & $0.3(0.6)$ & $1.3(0.2)$ & $30.7(17.2)$ & $0.7(1.2)$ & - & - \\
\hline Surgical (SICU) & $0.3(0.8)$ & $4.4(4.5)$ & $2.9(2.0)$ & $38.3(18.4)$ & $1.1(1.1)$ & - & $0.6(1.5)$ \\
\hline Total & $0.3(0.6)$ & $2.4(3.3)$ & $2.2(2.7)$ & $35.6(20.2)$ & $0.6(0.9)$ & - & $0.2(0.9)$ \\
\hline \multicolumn{8}{|c|}{ Perceived } \\
\hline Medical (MICU) & $0.3(0.5)$ & $1.2(1.8)$ & $1.6(1.8)$ & $6.8(3.9)$ & $0.3(0.7)$ & - & - \\
\hline Neurosurgical (NSICU) & $1.0(1.0)$ & $2.3(2.1)$ & $2.0(1.7)$ & $8.3(3.5)$ & $4.3(5.1)$ & - & $3.3(2.9)$ \\
\hline Surgical (SICU) & $0.1(0.4)$ & $1.4(1.5)$ & $1.6(1.3)$ & $6.1(3.7)$ & $0.7(1.0)$ & - & $0.1(0.4)$ \\
\hline Total $(N=20)$ & $0.4(0.6)$ & $1.5(1.7)$ & $1.7(1.5)$ & $6.8(3.6)$ & $1.1(2.3)$ & - & $1.0(1.8)$ \\
\hline
\end{tabular}

perceived number of times they performed each task during a typical 12-hour shift.

2.5. Procedures. Nurses were observed performing job tasks during the entire 12-hour shift. All observations were conducted from a distance to eliminate interference with normal care duties and to ensure medical care was not influenced. This also minimized the potential of hearing confidential information. Nurses informed patients under their care about the study with verbal consent being granted before the researcher entered the room to conduct observations. Three observers from the research team were trained to collect data and conducted the observations. An initial 12-hour observation was conducted with all 3 observers from the research team collecting data on a single nurse. This was done to ensure uniformity in data collection. The remaining observations were conducted with two observers including the study primary investigator.

Upon entering the study, all participants read and signed an IRB-approved consent form prior to completing any assessments. Participants were provided with written information detailing the purpose, data collection methods, and associated experimental procedures. At the end of each shift, the observed participant completed a short survey on the perceptions of lifting and transferring on a typical shift. The survey provided a list of patient handling tasks and required the nurse to provide a number documenting the amount of times during the shift the tasks are performed. For the entire shift, one member of the research team followed the nurse and documented the interaction with patients, medical equipment, and lift/transfer assist devices.
2.6. Data Analyses. Given the observational nature of the study, simple descriptive statistics (mean and standard deviations) were computed for the individual ICUs as well as overall. Small sample sizes and highly quantitative data observation limited the statistical analyses.

\section{Results}

3.1. Overall. Direct observation documented nurses manually handling patients and equipment an average of 72 times during a 12-hour shift. Lifting assist devices were utilized only $3 \%$ of the time when transferring or repositioning patients. The perception of the nurses resulted in lower estimates with a perceived average of 27 patient and equipment handling activities per 12-hour shift (see Figure 1).

\subsection{Patient Handling (Transferring and Repositioning)}

3.2.1. Objective Observation. The majority of patient handling involved repositioning of the patient in bed (see Table 2). Repositioning of patients was observed an average of 35 times during a 12-hour shift. When breaking down the repositioning tasks, the average number per 12-hour shift was 8 times of repositioning patient up in bed, 19 times of repositioning extremities, 1 time of repositioning patient laterally, and 7 times of turning patient on side. Transferring patient from the bed occurred relatively infrequently with an average of about 4 times per shift (2.4 to the chair, 2.2 out of bed to standing position, and 0.3 to wheelchair). There were no observations of transferring patient in and out of tub or shower but the nurses did transfer patients to/from the bathroom in the form of transfers to/from the toilet but it occurred infrequently 
TABLE 3: Frequency (number of times per shift) of medical equipment and furniture handling for the nurses in the three ICU units and overall (average and standard deviation).

\begin{tabular}{|c|c|c|c|c|c|}
\hline Unit & Push equipment & Pull equipment & Reposition furniture & Reposition equipment & Lift item from floor \\
\hline \multicolumn{6}{|c|}{ Direct observation } \\
\hline Medical (MICU) & $6.1(3.2)$ & $6.2(3.6)$ & $3.5(3.1)$ & $6.0(2.4)$ & $3.6(4.6)$ \\
\hline Neurosurgical (NSICU) & $3.0(2.6)$ & $3.0(2.6)$ & $2.3(2.5)$ & $4.3(2.0)$ & $1.7(2.9)$ \\
\hline Surgical (SICU) & $7.0(6.2)$ & $6.9(7.0)$ & $4.0(4.1)$ & $8.1(7.1)$ & $0.1(0.4)$ \\
\hline Total $(N=20)$ & $6.0(4.5)$ & $6.0(4.9)$ & $3.5(3.3)$ & $6.5(4.7)$ & $4.3(5.6)$ \\
\hline \multicolumn{6}{|c|}{ Perceived } \\
\hline Medical (MICU) & $6.1(5.7)$ & $2.3(1.9)$ & $5.0(3.5)$ & $4.7(3.0)$ & $11.4(9.9)$ \\
\hline Neurosurgical (NSICU) & $6.3(1.2)$ & $9.0(5.3)$ & $6.7(4.7)$ & $11.7(7.6)$ & $8.3(1.5)$ \\
\hline Surgical (SICU) & $5.7(4.3)$ & $5.6(4.4)$ & $3.6(3.3)$ & $4.9(3.9)$ & $0.3(0.5)$ \\
\hline $\operatorname{Total}(N=20)$ & $6.0(4.6)$ & $4.5(4.1)$ & $4.8(3.6)$ & $5.8(4.6)$ & $9.9(7.7)$ \\
\hline
\end{tabular}

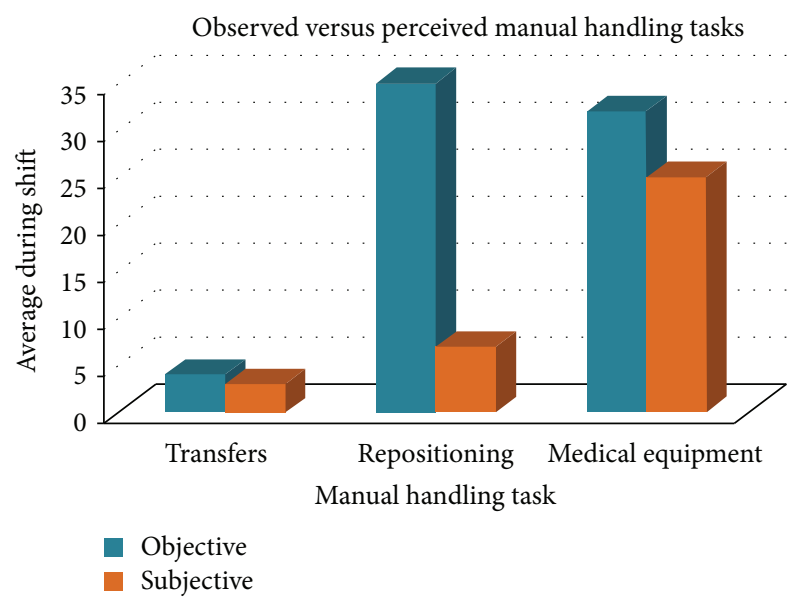

FIGURE 1: Comparison between the observed (objective) and the reported (perceived) for handling of patients (transfers and repositioning) and medical equipment.

over the duration of the shift (about 1 per shift). Transferring of patients was performed manually, with the limited use of lift assist devices. Lift assist devices were utilized only $3 \%$ of the time during patient handling activities.

3.2.2. Perception. A completely different picture of the handling activities was found based on nurse perception (Table 3). Repositioning of the patient in bed was the most frequent (about 7 times per shift) and transferring of patients in and out of bed was the least frequent at 3 times per shift (either to chair, wheelchair, or standing posture).

\subsection{Handling of Medical Equipment and Furniture}

3.3.1. Objective Observation. Nurses were observed to routinely handle medical equipment and furniture (see Table 3 ). Nurses pushed and pulled medical equipment about once an hour for the entire shift (about 12 times per shift) and repositioned equipment (e.g., lifting medical monitors to attach to beds or IV poles) about 6 times per shift. While not as frequent as handling medical equipment, moving furniture occurred routinely, about 5.5 times per 12-hour shift.

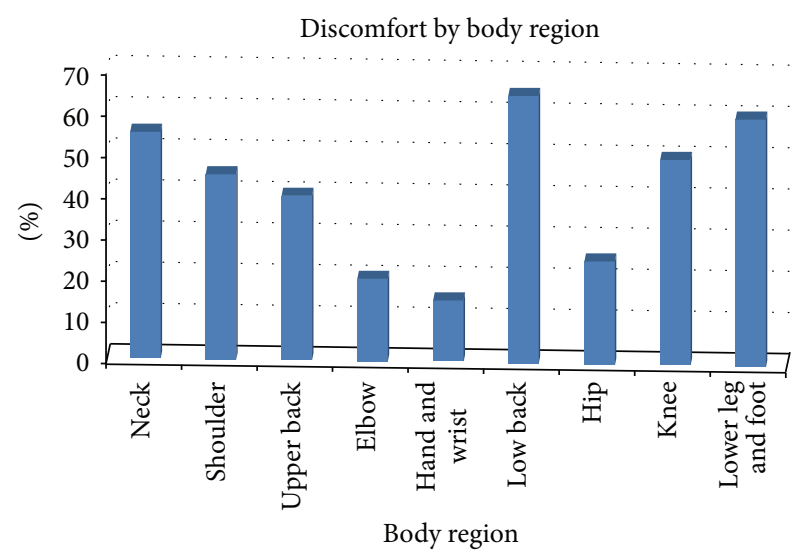

FIGURE 2: Discomfort in body regions as reported by survey completed by the nurses at the end of the shift.

Interestingly, nurses lifted items off the floor about 5 times per shift, with MICU and CICU nurses' lifting close to 10 items from the floor per shift.

3.3.2. Perception. Nurses were observed to routinely handle medical equipment and furniture (see Table 3 ). They pushed and pulled medical equipment about once an hour for the entire shift (about 12 times per shift). Nurses perceived to reposition equipment (e.g., lifting medical monitors to attach to beds or IV poles) about 6 times per shift. While not as frequent, moving furniture occurred routinely at about 3.5 times per 12-hour shift. The nurses also perceived that they routinely lifted items from the floor about 4 times per shift.

3.3.3. Body Discomfort. Based on the Symptom Survey completed by participants during the observations, the three most prevalent areas of pain experienced by nurses were low back pain, lower leg and ankle pain, and neck pain (see Figure 2). More than $65 \%$ of participants reported experiencing low back pain, with $45 \%$ reporting the pain as moderate to severe. Lower leg and ankle pain was reported by $60 \%$ of participants with $30 \%$ reporting the pain as moderate to severe. Fifty-five percent of participants reported neck pain and $15 \%$ of nurses experienced moderate to severe pain. Table 
TABLE 4: Body region discomfort (average and standard deviation) at the end of the shift for nurses in each of the ICU units and overall.

\begin{tabular}{lccccccccc}
\hline Unit & Neck & Shoulder & Elbow & Hand and wrist & Upper back & Low back & Hip & Knee & Lower leg and foot \\
\hline Medical (MICU) & $2.7(5.7)$ & $1.3(2.7)$ & $0.7(1.6)$ & $0.4(1.2)$ & $2.1(4.7)$ & $3.4(7.2)$ & $0.4(1.2)$ & $2.1(4.4)$ & $4.9(9.7)$ \\
Neurosurgical (NSICU) & $3.0(4.4)$ & $2.0(1.7)$ & $0.7(1.2)$ & $0.7(1.2)$ & $5.0(1.7)$ & $7.3(2.1)$ & $3.7(4.0)$ & $3.3(3.5)$ & $3.0(4.4)$ \\
Surgical (SICU) & $1.3(1.3)$ & $1.3(2.0)$ & $0.0(0.0)$ & $0.1(0.4)$ & $1.3(2.4)$ & $3.1(2.5)$ & $1.4(2.7)$ & $1.3(1.7)$ & $1.6(2.3)$ \\
Total (N = 20) & $\mathbf{1 . 9 ( 2 . 4 )}$ & $\mathbf{1 . 2 ( 1 . 6 )}$ & $\mathbf{0 . 4 ( 0 . 8 )}$ & $\mathbf{0 . 3 ( 0 . 8 )}$ & $\mathbf{2 . 0 ( 2 . 6 )}$ & $\mathbf{3 . 4 ( 3 . 2 )}$ & $\mathbf{1 . 2 ( 2 . 4 )}$ & $\mathbf{1 . 7 ( 2 . 0 )}$ & $\mathbf{2 . 7 ( 3 . 1 )}$ \\
\hline
\end{tabular}

4 provides a detailed breakdown of the average values for body discomfort. While most of the discomfort at the end of the shift would be considered to be mild, discomfort in the low back and legs was elevated (above 2.5 ). While only a casual inference can be made, the type of ICU may influence where the pain was felt-NICU had higher levels in back and lower extremity as a whole while MICU and SICU had pain predominantly in low back and lower legs.

\section{Discussion}

The objective of this study was to observe and quantify the frequency of handling tasks performed by nurses in hospital intensive care units and further evaluate nurses' perceptions of patient handling tasks performed. The results of the perceived patient handling survey were compared to the observed values documented by the research team. The age and gender distribution of the sample population reflected the nursing staff demographics at the University of Cincinnati Medical Center. Based on observations, nurses have a high prevalence of manual lifting of both patients and medical devices, but limited utilization of lift assist devices.

The objective results revealed that $14 \%$ of patient handling tasks involved patient transfers while repositioning accounted for the remaining $86 \%$ of patient handling related job tasks. Callison and Nussbaum [6] found that repositioning was dramatically less (16\%) and transferring between bed and chair was much higher (7\%) than what was found in the current study. One main difference in the relative percentage for the activities was that Callison and Nussbaum made an evaluation only over a 2-hour period rather than an entire 12hour shift. These objective frequencies were also consistent with evidence presented in a previous Garg study [4], which showed repositioning and patient transfers as top patient handling tasks performed by nurses. Majority of patient handling tasks involved repositioning of patients, with a primary focus on repositioning extremities (54\% of reposition tasks). Repositioning of the extremities may seem to be a trivial effort. However, lifting legs or arms of heavier (e.g., obese or morbidly obese) individuals would pose significant physical demands on the nurse. More strenuous repositioning activities were also routinely observed: repositioning of entire body up in the bed (23\% of the repositioning activities or 8 times per shift) and turning on the patient on their side (20\% of the repositioning activities or 7 times per shift). Lateral repositioning occurred infrequently (1 time per shift). The high frequency of repositioning likely resulted from a combination of patient requesting repositioning (e.g., repositioning of extremities), patient migration (e.g., repositioning entire position up the bed), and policies of requiring nurses to turn limited-mobility patients on sides every 2-hours (e.g., turning patient on side). As a result, the majority of patient handling tasks were focused on patient comfort and ease (e.g., correcting patient position due to migration down the bed) and ease of administering care (e.g., collecting vital information, responding to medical concerns, etc.). Warming and associates [15] utilized self-reported logbooks to document patient handling activities. These researchers have documented nurses completing patient transfers and repositioning tasks about 6 times and 7 times per shift, respectively. The different distribution between the current study and the Warming study may have resulted from different hospital units.

The observed patient handling frequencies may have been a direct reflection of the units observed. Nurses were recruited from intensive care units (MICU, NSICU, and SICU). The majority of the patients in these units required assistance with minimal mobility. The focus of all three units was to stabilize the patient to the medical level, which would allow them to be transferred to a regular room. With the main goal being to prevent further medical complications associated with lack of mobility, frequent repositioning was required. Further, nurses were assigned to no more than 3 patients and were required to reposition patients at least once every 2 hours.

In this current study, lift assist devices were utilized during 3\% of observed patient handling tasks and this is consistent with the Garg study [4], which reported assistive devices being utilized less than $2 \%$ of the time. The underlying reasons for the low usage of lift assist devices were not investigated in the current study given that the focus was on quantifying the handling activities. Use of bed sheets to reposition and move patients was routinely observed but was not documented as a lift assist device. Bottom line, lifting assist devices were rarely utilized in the intensive care units when handling patients.

Handling tasks in the ICUs also included frequent handing of medical equipment, in-room furniture, and maneuvering objects such as trash receptacles, tubs of water for patient cleaning needs, and required waste handling containers. Equipment was handled by nurses an average of 6 times during a 12-hour shift while furniture was repositioned an average of 5 times per shift. In addition, nurses were found to routinely push, pull, and lift equipment to accomplish patient care tasks. Handling equipment and other patient care objects accounted for over $38 \%$ of observed handling tasks. Moving furniture in the room was a routine activity (about 4 times a shift), accounting for about 15\% of handling 
activities. One potential factor that leads to routine moving of furniture could be the physical layout of the ICU rooms that are designed with a focus on providing care to patient in the bed. Family members and visitors used the furniture, moving them close to the bed, which then had to be moved away from the bed by the nurse when medical treatment was completed. Medical equipment was routinely pushed or pulled to and from the patient's bedside (about 8 times per shift) when providing care. One of the more interesting observed activities was lifting equipment or items (e.g., monitors, medical equipment) from the floor to the bed, which occurred frequently ( 6 times per shift or $16 \%$ of equipment handling tasks). These tasks may pose risk of low back injuries but have not been identified in previous studies evaluating the handling activities of nurses. Further investigation is needed to understand the biomechanical stresses associated with routinely lifting equipment or items from the floor in hospital settings.

The perceptions of nurses about the handling activities were very different from the observed frequencies, especially for patient repositioning. Overall, the number of handling activities was perceived to be $50 \%$ lower than the actual values. The objective frequency of patient repositioning was observed 5 times more often than the perceived frequency. This significant difference may be a result of nurses viewing lifting limbs as interactions rather than repositioning. The exact reason for the difference would require further investigation. A smaller difference between the observed and the perceived was found for patient transfers ( 5 observed versus 4 perceived per shift). The perceptions of handling medical equipment and furniture in the room were much closer to reality. Handling medical equipment was perceived to be slightly lower than observed frequencies ( $88 \%$ of observed), while moving furniture was perceived to happen more often (about 37\%).

Nurses reported high levels of discomfort at the start of the shift. Discomfort survey results were reviewed for nurses reporting at a level of 1.0 or more and also separated into four categories $(0=$ None, $1-4=$ Mild, $5-8=$ Moderate, and 9-10 $=$ Severe). The top three areas for discomfort were low back $(65 \%)$, lower leg $(60 \%)$, and neck pain $(55 \%)$. Moderate to severe low back pain was reported at $45 \%$ and $30 \%$ for both moderate to severe neck pain and lower leg and ankle pain. Fifty-five percent of participants reported neck pain and $15 \%$ of nurses experienced moderate to severe pain. Many studies [16-18] have reported high levels of discomfort for lower back pain and it likely resulted from the physically demanding tasks performed routinely by the nurses. Pompeii and associates [19] reported that patient handling injuries were associated with repositioning patients in bed, pulling patients up in bed, and catching falling patients. The high level of repositioning observed in this study may be potentially linked to the high percentage of lower back discomfort reported by study nurses. This may have been further exacerbated due to the maneuvering and lifting of medical equipment, particularly from the floor. Further, nurses in these ICU locations rarely had the opportunity to sit during their 12hour shift as they were constantly performing patient checks, entering information into medical charts using a stand-up computer stations, and then performing additional patient handling tasks. Some were even observed eating their lunches standing up between patient care activities. This lack of opportunity to sit or rest may be associated with the high levels of lower leg and ankle pain. However, this observation is worth further investigation. Observing the discomfort levels by the four identified categories provided valuable information. Further study may lead to determining the association with particular patient handling tasks leading to high levels of discomfort. Linking the exact handling task to the outcome of discomfort levels could provide more details about safe patient handling intervention mechanisms.

4.1. Study Limitations. Nurses were observed for a single 12-hour shift for one adult hospital, resulting in potential sampling bias due to day-to-day variability for a given nurse, a single safety culture, and adult patients. While there was only one day for each subject, 12 hours of observation provided a good level of confidence that handling activities were accurately quantified. Further, sampling nurses on different days of the week also minimized the bias potentially due to cyclical patient levels. In all, the current study provides an objective estimate of the handling activities for nurses who work in intensive care units in an adult hospital.

While the data collection was intense (e.g., 12 hours of observation time for each subject), the number of subjects was on the low side and certainly limited the data and statistical analyses. Observing a larger sample population and including more hospitals and units within the hospitals would improve generalizability of the results. However, the results do provide a picture of the handling activities including patients, medical equipment, and furniture.

Finally, the assessment relied upon identification of the activities and not the actual biomechanical stress being placed on the nurses when performing the activities. The current results represent the first phase of understanding the stresses that are placed on the nurses and potentially lead to MSDs. Future studies need to follow up and estimate the continuous biomechanical loads being placed on the nurses throughout the shift.

\section{Conclusion}

The current study revealed that transferring of patients occurred during a 12-hour shift (about 4 times per shift) but less frequent than repositioning of the patient (about 35 times per shift). Moving medical equipment and furniture was also routinely done, 16 times and 5 times per shift, respectively. Results also indicated that there was a large mismatch between nurse perception of the handling of patients and medical equipment and reality of what was observed. In general, nurses underestimated the amount of lifting performed during a 12-hour shift. The mismatch between reality (directly observed) and perception (subjective estimate) may be a potential underlying factor for the lack of usage of lift devices as these devices were utilized in less than $2 \%$ of the transfer or repositioning tasks. It may be that the nurse underestimated the risk to manual handling and performed more risky lifting (e.g., no usage of lift assist devices). 


\section{Conflict of Interests}

The authors declare that there is no conflict of interests regarding the publication of this paper.

\section{References}

[1] Bureau of Labor Statistics, Nonfatal Occupational Injuries and Illnesses Requiring Days Away from Work, 2009, US Department of Labor, USDL, 2010.

[2] A. Nelson, G. Fragala, and N. Menzel, "Myths and facts about back injuries in nursing," The American Journal of Nursing, vol. 103, no. 2, pp. 32-41, 2003.

[3] K. G. Davis and S. E. Kotowski, "Prevalence of musculoskeletal disorders in healthcare providers in hospital, long term care facilities and home healthcare: a comprehensive review," Human Factors. In press.

[4] A. Garg, B. D. Owen, and B. Carlson, "An ergonomic evaluation of nursing assistants' job in a nursing home," Ergonomics, vol. 35, no. 9, pp. 979-995, 1992.

[5] T. R. Waters, "When is it safe to manually lift a patient?" The American Journal of Nursing, vol. 107, no. 8, pp. 53-58, 2007.

[6] M. C. Callison and M. A. Nussbaum, "Identification of physically demanding patient-handling tasks in an acute care hospital," International Journal of Industrial Ergonomics, vol. 42, no. 3, pp. 261-267, 2012.

[7] G. T. Holman, K. J. Ellison, S. Maghsoodloo, and R. E. Thomas, "Nurses' perceptions of how job environment and culture influence patient handling," International Journal of Orthopaedic and Trauma Nursing, vol. 14, no. 1, pp. 18-29, 2010.

[8] S. B. Randall, W. J. Pories, A. Pearson, and D. J. Drake, "Expanded Occupational Safety and Health Administration $300 \log$ as metric for bariatric patient-handling staff injuries," Surgery for Obesity and Related Diseases, vol. 5, no. 4, pp. 463468, 2009.

[9] A. Myers, R. C. Jensen, D. Nestor, and J. Rattiner, "Low back injuries among home health aides compared with hospital nursing aides," Home Health Care Services Quarterly, vol. 14, no. 2-3, pp. 149-155, 1993.

[10] A. Retsas and J. Pinikahana, "Manual handling activities and injuries among nurses: an Australian hospital study," Journal of Advanced Nursing, vol. 31, no. 4, pp. 875-883, 2000.

[11] Y. B. Yip, "A study of work stress, patient handling activities and the risk of low back pain among nurses in Hong Kong," Journal of Advanced Nursing, vol. 36, no. 6, pp. 794-804, 2001.

[12] D. R. Smith and P. A. Leggat, "Musculoskeletal disorders among rural Australian nursing students," Australian Journal of Rural Health, vol. 12, no. 6, pp. 241-245, 2004.

[13] A. Vasiliadou, G. G. Karvountzis, A. Soumilas, D. Roumeliotis, and E. Theodosopoulou, "Occupational low-back pain in nursing staff in a Greek hospital," Journal of Advanced Nursing, vol. 21, no. 1, pp. 125-130, 1995.

[14] B. Owen and A. Garg, "Assistive devices for use with patient handling tasks," in Advances in Industrial Ergonomics and Safety II, B. Das, Ed., pp. 585-592, Taylor \& Francis, Philadelphia, Pa, USA, 1990.

[15] S. Warming, D. H. Precht, P. Suadicani, and N. E. Ebbehøj, "Musculoskeletal complaints among nurses related to patient handling tasks and psychosocial factors-based on logbook registrations," Applied Ergonomics, vol. 40, no. 4, pp. 569-576, 2009.
[16] P. Harber, E. Billet, M. Gutowski, K. SooHoo, M. Lew, and A. Roman, "Occupational low-back pain in hospital nurses," Journal of Occupational Medicine, vol. 27, no. 7, pp. 518-524, 1985.

[17] R. M. Lynch and A. Freund, "Short-term efficacy of back injury intervention project for patient care providers at one hospital," American Industrial Hygiene Association, vol. 61, no. 2, pp. 290294, 2000.

[18] I. Bejia, M. Younes, H. B. Jamila et al., "Prevalence and factors associated to low back pain among hospital staff," Joint Bone Spine, vol. 72, no. 3, pp. 254-259, 2005.

[19] L. A. Pompeii, H. J. Lipscomb, A. L. Schoenfisch, and J. M. Dement, "Musculoskeletal injuries resulting from patient handling tasks among hospital workers," American Journal of Industrial Medicine, vol. 52, no. 7, pp. 571-578, 2009. 


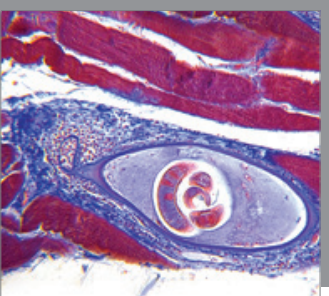

Gastroenterology

Research and Practice
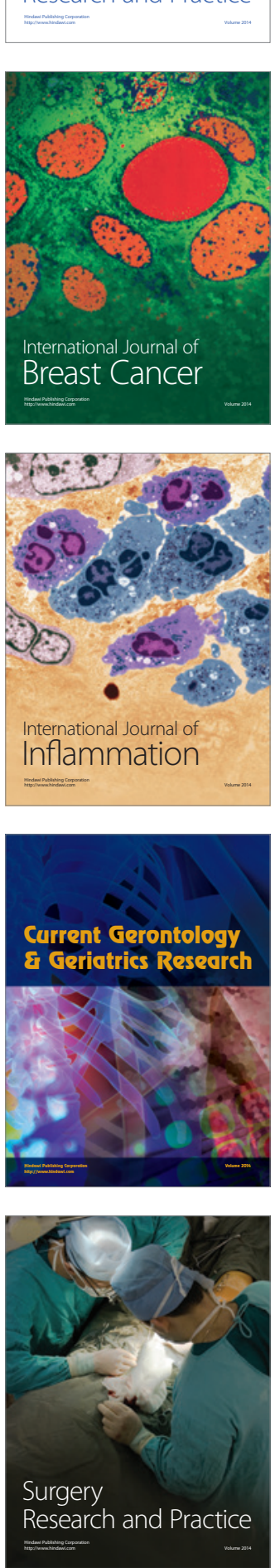

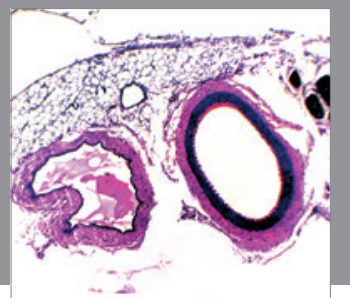

International Journal of Hypertension
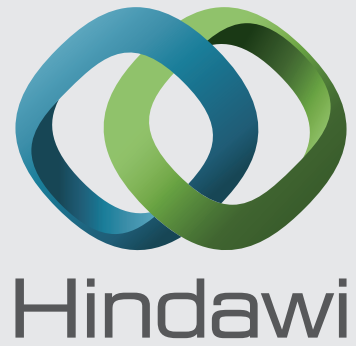

Submit your manuscripts at http://www.hindawi.com
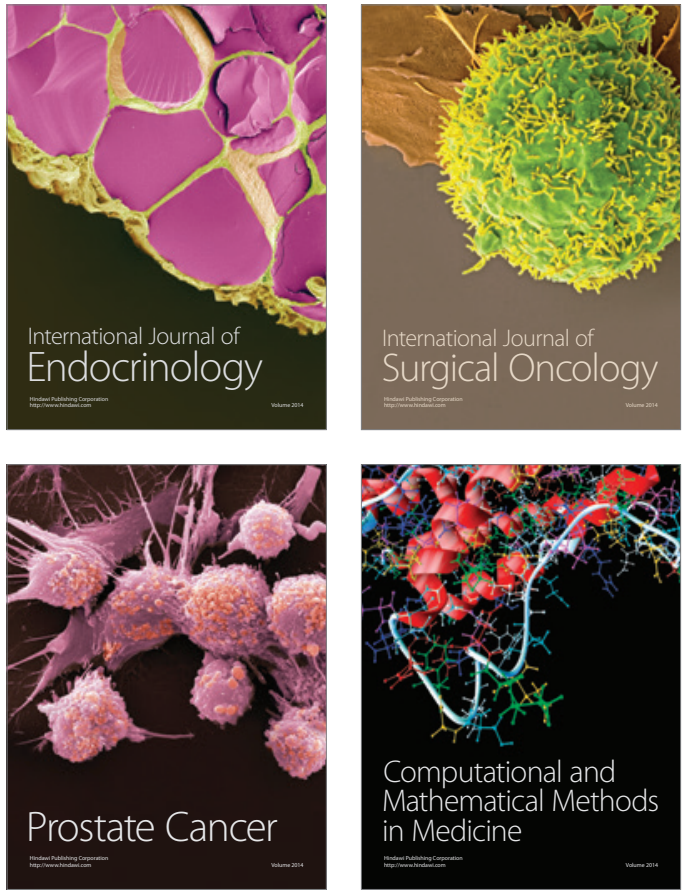
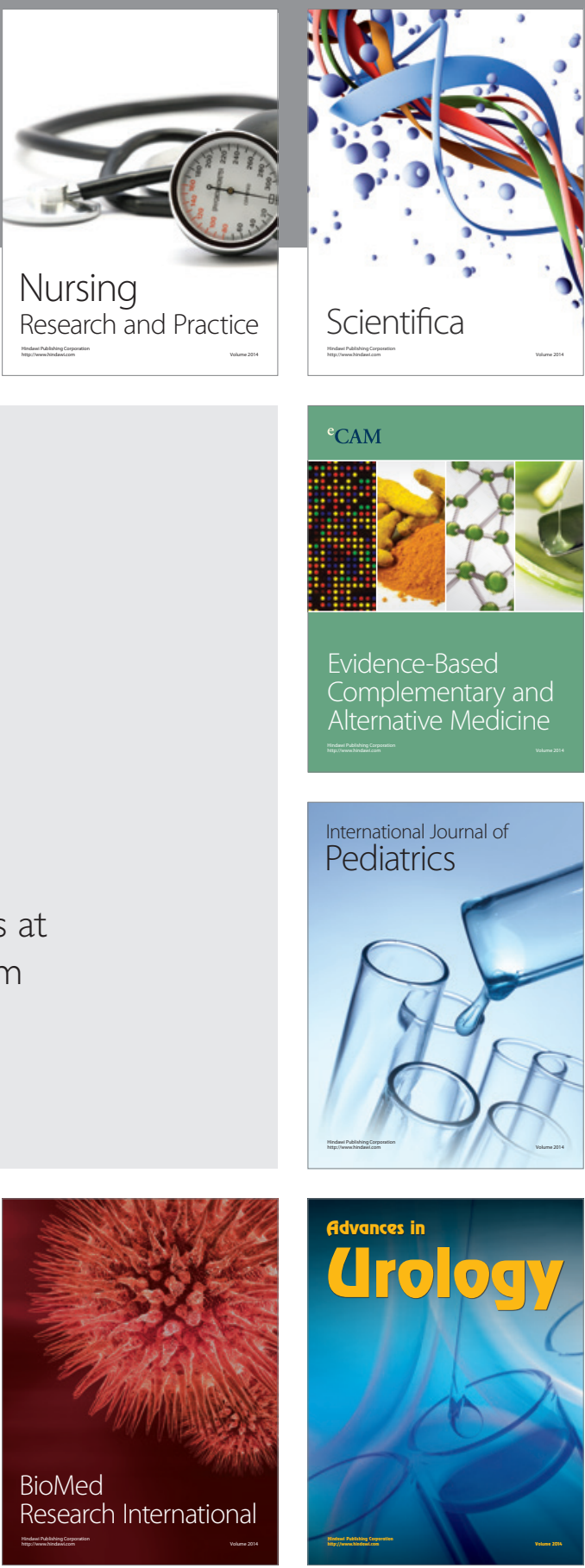

Nursing

Research and Practice

Scientifica

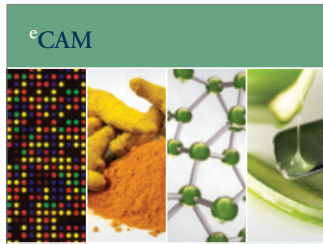

Evidence-Based

Complementary and Alternative Medicine
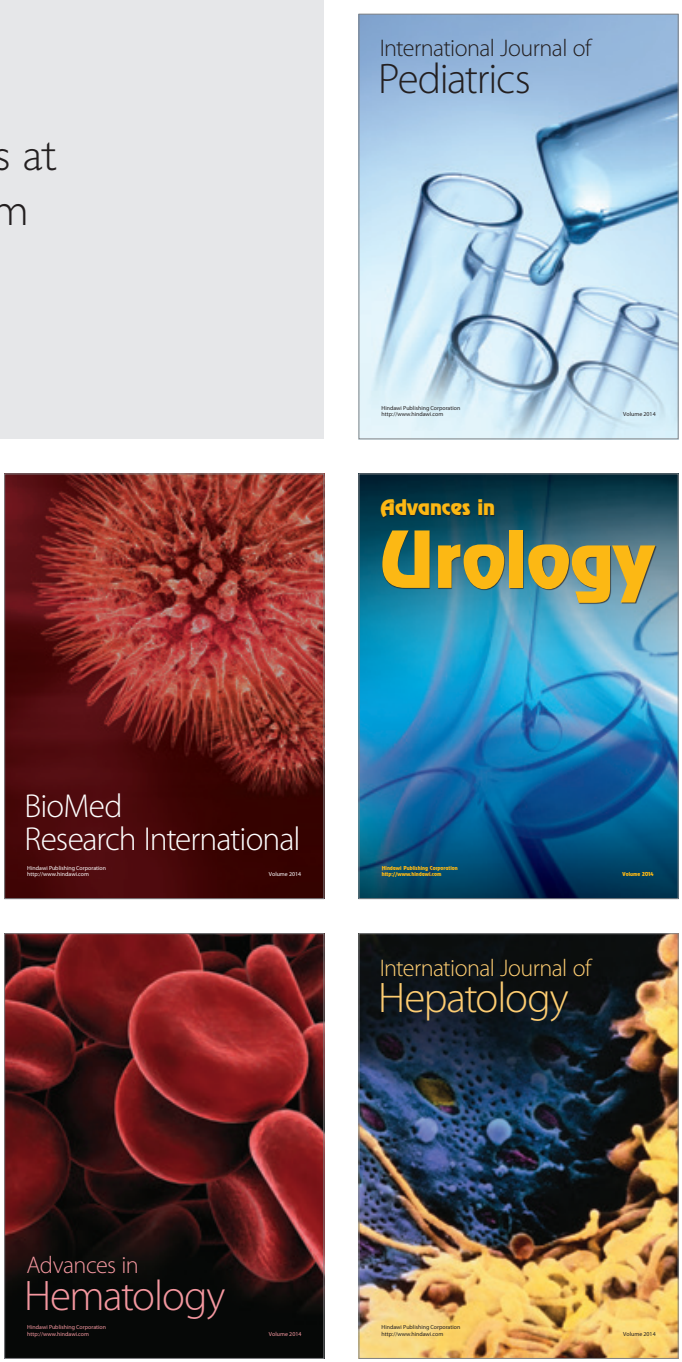